\title{
Feasibility Study on the Assessment of Auditory Sustained Attention through Walking Motor Parameters in Mild Cognitive Impairments and Healthy Subjects
}

\author{
L. Fiorini, M. Maselli**, E. Castro, S. Tocchini, M. T. Sportiello, C. Laschi, Senior, IEEE, \\ F. Cecchi, Member, IEEE, F. Cavallo, Member, IEEE
}

\begin{abstract}
Dementia and other cognitive disorders will affect more than 35 million people worldwide. Over the last years, cognitive training tools were used to improve the brain functioning, thus to slow down the cognitive decline. Recently, research studies have demonstrated that aerobic exercise could play an important restorative role toward cognitive impairments. Therefore, the aim of this work is to present an innovative sensorized approach which combine aerobic exercise and traditional cognitive tools for daily training.

This feasibility study consists of two parts, the first part aims to design, and develop the ICT device and the experimental protocol, whereas the latter one aims to demonstrate that the output of the sensorized system could be correlated with traditional test in measuring the same cognitive domains.

The proposed system was tested with fifteen subjects (11 healthy controls and 4 subjects affected by Mild Cognitive Impairments) in a controlled environment. The results suggest a good correlation between the two approaches.
\end{abstract}

\section{INTRODUCTION}

Worldwide, in the past decades, the human lifespan has been lengthened; the cause of this phenomenon could be recovered in a combination of decreasing fertility and increasing life expectations [1]. The life lengthening is increasing the impact of typical aging diseases that nowadays are involving a significant part of the population. One of the most significant diseases among the senior citizens is dementia, which affects more than 35 million people worldwide [2]. Indeed, dementia is the prominent chronic disease in the older age, and one of the most remarkable cause of disability [3]. According to the World Health Organization (WHO), in 2010, the global societal cost of dementia was assessed to be US\$ 604 billion. For this reasons, the importance of successful aging, intended as a "low probability of disease and disease-related disability, high cognitive and physical functional capacity, and active engagement with life" [4] has been deeply intensified during recent years.

*Research supported by DAPHNE project (REGIONE TOSCANA PAR FAS 2007-2013, BANDO FAS SALUTE 2014, CUP J52I16000170002).

L.Fiorini, E.Castro, M.Maselli, C.Laschi, F.Cecchi, F.Cavallo are with the BioRobotics Istitute of Scuola Superiore Sant'Anna, Viale Rinaldo Piaggio 34, 56025 Pontedera (PI), IT (phone: 0039-0587-672158; email:1.fiorini@santannapisa.it, m.maselli@santannapisa.it.

S. Tocchini and M. Timpano Sportiello, Jr., are with USL Nordovest Toscana, Laboratory of Neuropsychology, Pontedera, Italy. E-mail: stefania.tocchini@hotmail.it, marco.timpano@uslnordovest.toscana.it.

**Corresponding Author: (phone: +39 050 883078; fax: +39 050 883497; e-mail: m.maselli@santannapisa.it).
Cognitive training (CT) involves of a variety of exercises aimed to help improve brain functioning. Even if results are not definitive, cognitive trainings have been demonstrated to be successful in reducing mental declines in elderly with cognitive impairment and dementia, especially at an early stage [5],[6]. Moreover, CT has been used to improve cognitive performances and to delaying daily functions difficulties in healthy elderly too [7],[8]. Typically, cognitive training is performed by classic pen-and-paper exercises, by neuropsychological software or by video-games [9]. Nevertheless, recently, cognitive functioning has been proved to be improved by physical training too, even if the role of exercise on brain is still unclear [10-13].

As state from literature, the protective and restorative role of physical activity toward cognitive impairment and dementia has been particularly highlight for aerobic exercise [14],[15]. According to this results, the cognitive training protocols involving a mixed paradigm (cognitive and physical exercise) has begun to been developed, reporting encouraging results [16].

Therefore, in line with such research-line, this study aims at developing a tool able to combine the possibility to perform a cognitive and an aerobic physical training at the same time, in order to verify the effect of its utilization on cognitive performances. To fulfil this goal, a reproduction of a standardized neuropsychological subtest, taken from the TAP battery [17], was developed and enriched, creating SmartWalk system. The cognitive training involves the attention domain, while the exercise training is an aerobic one (walking activity).

In this paper, we describe the SmartWalk system and then we present a feasibility study aims at demonstrate that SmartWalk can be used in substitution of the TAP subtest. To achieve this goal, firstly, we compare the scores in order to verify if the SmartWalk system could be correlated with a parallel version of the traditional TAP subtest. Then, we aim at investigating whether the SmartWalk system is able to cluster the performance of healthy and Mild Cognitive Impairment (MCI) subjects in order to reflect the clinical assessment.

\section{METHODOLOGY}

This section mainly describes the two cognitive tools (TAP and SmartWalk), the experimental protocol, the participants, and the analysis performed to accomplish the desired outcomes. 


\section{A. Cognitive tools}

Traditional test: TAP test - The "Test for Attentional Performances" (TAP) was originally developed for the evaluation of attentional deficits in patients with cerebral lesions by Zimmerman et al. [17]. The test battery is a computerized technique. The battery is composed by various subtests able to assess various aspects of the attentional domain, such as alertness, divided attention, vigilance, neglect, visual search, working memory, inhibitory process, focused attention.

In the vigilance subtest, four tasks with different stimuli are presented in order to assess sustained attention. The tasks are unimodal (an acoustic task and two visual tasks) and bimodal (visuo-acustic task) in order to evaluate if one or both the sensorial channels result impaired.

The output measures that neuropsychology use to assess the TAP subtest for auditory sustained attention are listed in Table. I.

In the acoustic task, reproduced in this study with a parallel version, a sequence of alternated high and low tone was presented to the subject. The subject had to detect an irregularity in the sequence (same tone repeated twofold). As soon as he/she detected it, he/she had to click the mouse. A software collected the response.

SmartWalk test - one of the goals of this study is to combine aerobic activity with traditional cognitive tolls. SmartWalk test has been designed to fuse walking activity ad TAP test for auditory sustained attention.

SmartWalk system is composed of a wearable inertial sensor placed on the dominant foot (SensorFoot) and a software which collect the data (Fig.1.). SensorFoot V2 (Fig.1.a) is able to collect a 9-axis inertial system (3-axis accelerometer, a 3-axis gyroscope and a 3-axis magnetometer) at a frequency of $50 \mathrm{~Hz}$. The data is filtered on-board with a fourth-order low-pass digital Butterworth filter with a $5 \mathrm{~Hz}$ cut-off frequency. The core of this device is represented by the iNEMO-M1 system on board (STMicroelectronics, Italy) with a Cortex-M3 family microcontroller [18]. SensorFoot V2 has a small battery that supplies the system. Data are collected and transmitted via Bluetooth protocol to the software module installed on a computer. The software module is developed with Visual

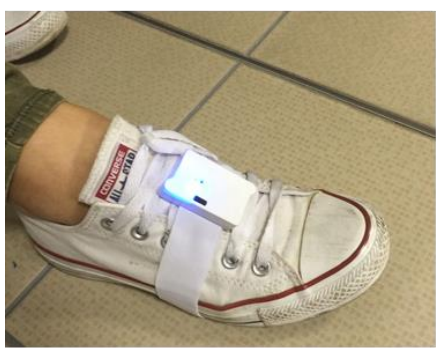

(a)

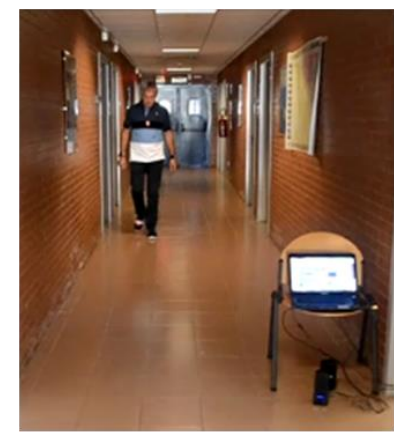

TABLE I. OUTPUT MEASURES OF TRADITIONAL AUDITORY SUSTAINED ATTENTION TEST.

\begin{tabular}{|l|l|}
\hline Scores & Description \\
\hline Correct & $\begin{array}{l}\text { Number of changes in the audio track correctly } \\
\text { identified. The user properly react clicking the } \\
\text { mouse by a time-window of 1500 ms. }\end{array}$ \\
\hline Delay & $\begin{array}{l}\text { Number of changes in the audio track correctly } \\
\text { identified. The user properly react clicking the } \\
\text { mouse by a time-window of 2500 ms. }\end{array}$ \\
\hline Omitted & $\begin{array}{l}\text { Number of changes in the audio track not } \\
\text { identified }\end{array}$ \\
\hline False allarms & $\begin{array}{l}\text { Number of changes identified in absence of } \\
\text { changes in the audio track }\end{array}$ \\
\hline Mean & Mean "reaction" time of correct answers \\
\hline Median & Median "reaction" time of correct answers \\
\hline Standard Deviation & $\begin{array}{l}\text { Standard deviation of "reaction" time of correct } \\
\text { answers }\end{array}$ \\
\hline
\end{tabular}

Studio 2013 (c\# language) and it is able (i) to collect and store SensorFoot V2 data, (ii) to autonomously administrate the exercise and (iii) to reproduce the audio track for testing the sustained attention (the same of TAP subtest).

During this test, the participant was asked to wear the SmartFoot on the dominant foot. After the initial guidelines administrated by SmartWalk software, the user was asked to walk at their own "natural velocity" for the entire test. Every time he/she observed a variation in the auditory sequence, he/she had to kick (Fig.1.b).

\section{B. Protocol}

The main goal of this work is to investigate whether the two cognitive tools are correlated in measuring the same cognitive domains. For this reason, participants were asked to perform both tests in two separate sessions in order to compare correspondent output measures. The two tests were assessed in a random order. Each test lasts 30 minutes. A psychologist and a technician assisted the tests in order to intervene in case of necessity. The data were acquired and stored by a computer.

\section{Participants}

Fifteen voluntary subjects ( 7 male, 8 female) participated on purpose in this study. Particularly, 11 participants were cognitive healthy and they aged from 16 to 65 years old.
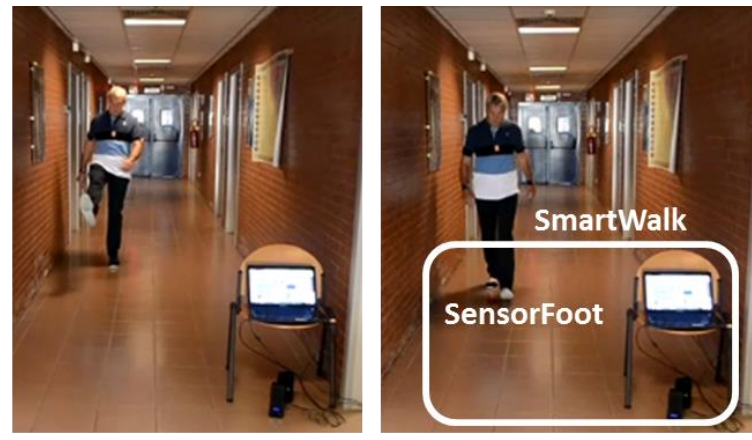

(b)

Figure 1. (a) SensorFoot, (b) a participant is performing SmartWalk test, the participantis walking at constant speed, as soon as he identified a change in the audio track he kicks and then he continues to walk. 
Whereas, a sample cohort of 4 participants were over 65 years old and they suffer from MCI. The diagnosis was made by expert clinicians according to Petersen criteria [19] for MCI. In particular: (i) the subjects suffered from a cognitive impairment not normal for their age; (ii) the subjects were not demented; (iii) the change in cognition did not cause a significant impairment in functional activities. An additional inclusion criterion was the possibility to walk continuously for a total of $30 \mathrm{~min}$.

Among tested subjects, all participants were right hand and the right foot was the dominant one. Participants completed the experimental session at the BioRobotics Institute of Scuola Superiore Sant'Anna (Pontedera, Italy). The protocol of the study was approved by the Ethics Committee of the Scuola Superiore Sant'Anna, Pisa. Written informed consent was obtained from all the participants before starting the tests. For young adults $(<18$ years old $)$ the informed consent was signed by one parent.

\section{Data analysis}

The SmartWalk data were off-line analysed with Matlab 2015 software (MathWorks Inc., Natick, MA, USA). Firstly, data was processed in order to recognize the events "kick" and the output measures. Then, they were compared with the measures obtained with the traditional TAP subtest.

\section{a) SmartWalk Data Pre-processing}

Data from 3-axial accelerometer $\left(a_{x}, a_{y}, a_{z}\right)$ was aggregate to compute the module as:

$$
\mathrm{M}(\mathrm{t})=\sqrt{\mathrm{a}(\mathrm{t})_{\mathrm{x}}{ }^{2}+\mathrm{a}(\mathrm{t})_{\mathrm{y}}{ }^{2}+\mathrm{a}(\mathrm{t})_{\mathrm{z}}{ }^{2}} \quad \forall t \in T
$$

Where $\mathrm{T}$ is the total time series and $\mathrm{t}$ is a specific instant of time. The event "kick" had been revealed as soon as M (Eq.1) was major of a threshold which was tuned on the personal gait. Then, the temporal "kick" sequence was synchronized with the auditory track by means of timestamp.

Same as in the traditional subtest, we computed the subtest scores in order to adequately compare the two approaches. In this feasibility study, we adapted the threshold "correct/delay/false alarms" scores according to the walking exercise. Summarizing, the final scores were assigned considering the $\Delta \mathrm{t}$ between the "kick" event and the sound variation as (i) $\Delta \mathrm{t}<2500 \mathrm{~ms}$ the "kick" was considered "Correct". (ii) $2501<\Delta \mathrm{t}<5000 \mathrm{~ms}$ the "kick" was considered "Delay". (iii) $\Delta \mathrm{t}>5001 \mathrm{~ms}$ the kick was considered "false alarms".

At the end of the analysis, all the "correct/delay/false alarms" events were counted and the statistic on the correct answer were computed. At the end of this process, for each participant, we had two sets of scores, one for the TAP subtest and one for the SmartWalk test.

\section{b) Comparison of the two methods}

In order to achieve the first goal, the two sets of output measures were statistically analyzed in order to verify the correlation between the two tests. Particularly, Pearson coefficient (r) was computed for correspondent scores to verify whether they were correlated or not. Additionally, t-
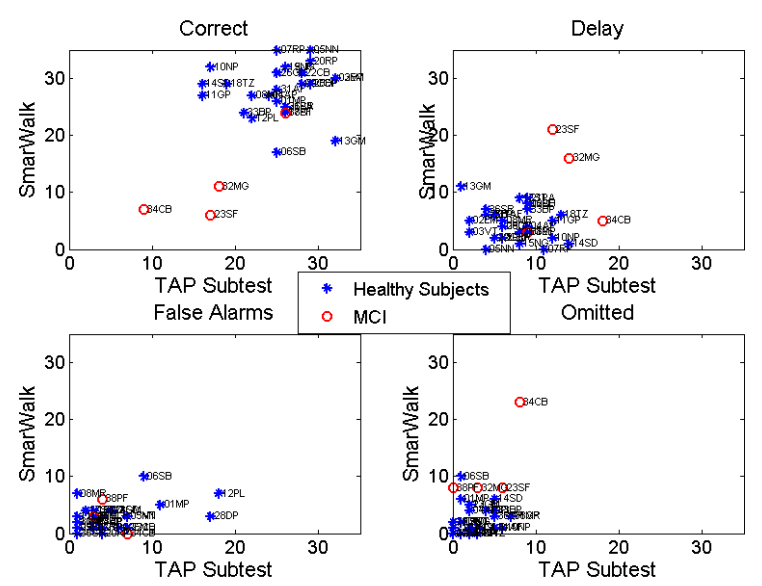

Figure 3. Scatter plot of output measure in TAP subtest and Smartwalk system for Correct, Delay, Omitted and False Alarms. The red circle are the MCI subjects and the blue stars are the healthy subjects

test was performed to verify if each couple of scores came from the same population.

As regard the second goal, in order to investigate whether the system is able to cluster the participants' performance according with the clinical cohort, the SmartWalk scores were normalized and reduced by Principal Components Analysis (PCA). Then, according with Kaiser rule, we considered only components with eigenvalues greater than 1 . Then K-Means (Euclidian distance, 2 replicates) was applied to group the participants according with SmartWalk results. Silhouette value [20] was computed to assess the cluster validity and to quantitatively analyse the clustering process. A higher value indicates that the clusters were compact and well separated.

\section{RESUlT AND DISCUSSION}

The aim of this research is twofold. Firstly we aim to compare the results of the two approaches and then we investigate whether the SmartWalk performance could reflect the clinical assessment. In this section the results are reported and discussed.

As regard the comparison between the two tests, the results show a high significant $(\mathrm{p}<0.05)$ correlation for the "correct" and "omitted" scores $(\mathrm{r}=0.73$ and $\mathrm{r}=0.59$ respectively). Also the "mean" and the "median" time are significant correlated $(\mathrm{r}=0.59$ and $\mathrm{r}=0.70$ respectively). These results are in line with the scatter plots depicted in Fig. 3. From a visual inspection, "correct" score shows the same outputs: if a participant obtained low score in the traditional test, he/she obtained low score also in the SmartWalk test. In is worth to mention that "Omitted" and "Correct" scores are considered by neuropsychologist to be the most significant measures in the traditional subtest. Because of that, the results of our comparison underline that the SmartWalk approaches could be used to assess auditory sustained attention. Additionally, Fig. 3 reports the output measures according with the clinical cohorts (healthy or MCI subject), it is also important to notice that, these measures could group the two cohorts according with the performance. 
Additionally, the t-test underlines that SmartWalk scores and the parallel version of traditional TAP subtest scores, concerning "correct", "delay", "omitted" and "false alarms" categories, belongs to the same populations $(p>0.05)$, which also means that the results were independent from the typology of tests. On the contrary, the "mean", "median" and "standard deviation" scores belong to different populations $(\mathrm{p}<0.05)$. These measures describe the "Reaction Time" which is mainly correlated with the typology of the tests. "Click a mouse" or "Kick" are two very different motor tasks, so the results are correlated but different.

As regard the cluster analysis, we select only the first two Principal Components (PCs) according with Kaiser rule. As reported in Fig.4, the blue stars represent the healthy population, whereas the red stars represent the MCI populations. From a visual inspection, the two cohorts seem to be grouped in the PCA space. K-means method was thus applied to confirm the evidence of clusters. The silhouette index was equal to 0.8086 , this high values confirms that the clusters were compacted and well-separated. Additionally, as depicted in Fig. 4, the participants seem to be correctly grouped according with the clinical group, except for participant $38 \mathrm{PF}$ which is on the edge between the two clusters (black and green circle).

\section{CONCLUSION}

The primary goal of this feasibility study is to compare the traditional subtest with our SmartWalk system that includes walking activity. The results suggest a good correlation between the two approaches. Future research should be focused to increase the number of participants to corroborate the study. Furthermore, usability study should be planned to estimate whether SmartWalk system could be used in a daily cognitive training at users' home.

\section{ACKNOWLEDGMENT}

The authors thank the clinical team of Laboratory of Neuropsychology of Pontedera (USL Toscana nord ovest) for their precious clinical support during the protocol definition and during the experimentation phase.

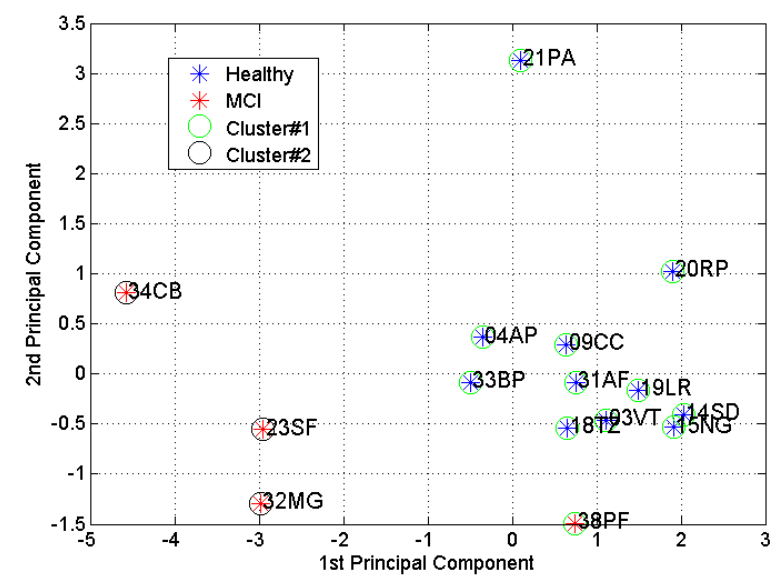

Figure 4. SmartWalk performance in the PCA space. The red and blue stars represent the clinical group, whereas the green and the black circle represent the K-Means results.

\section{REFERENCES}

[1] Nearly 27 million people aged 80 or over in the European Union, September 29, $2016 . \quad$ [Online] Available: http://ec.europa.eu/eurostat/documents/2995521/7672228/329092016-AP-EN.pdf/4b90f6bb-43c1-45ed-985b-dfbe9564157a. Accessed: Oct. 2016

[2] Prince, M., Bryce, R., Albanese, E., Wimo, A., Ribeiro, W., \& Ferri, C. P. (2013). The global prevalence of dementia: a systematic review and metaanalysis. Alzheimer's \& Dementia, 9(1), 63-75.

[3] Wimo, A., Jönsson, L., Bond, J., Prince, M., Winblad, B., \& International, A. D. (2013). The worldwide economic impact of dementia 2010. Alzheimer's \& Dementia, 9(1), 1-11.

[4] Rowe, J. W., \& Kahn, R. L. (1997). Successful aging. The gerontologist, 37(4), 433-440.

[5] Sitzer, D. I., Twamley, E. W., \& Jeste, D. (2006). Cognitive training in Alzheimer's disease: a meta- analysis of the literature. Acta Psychiatrica Scandinavica, 114(2), 75-90.

[6] Jean, L., Bergeron, M. È., Thivierge, S., \& Simard, M. (2010). Cognitive intervention programs for individuals with mild cognitive impairment: systematic review of the literature. The American Journal of Geriatric Psychiatry, 18(4), 281-296.

[7] Rebok, G. W., Ball, K., Guey, L. T., Jones, R. N., Kim, H. Y., King, J. W., ... \& Willis, S. L. (2014). Ten- year effects of the advanced cognitive training for independent and vital elderly cognitive training trial on cognition and everyday functioning in older adults. Journal of the American Geriatrics Society, 62(1), 16-24.

[8] Reijnders, J., van Heugten, C., \& van Boxtel, M. (2013). Cognitive interventions in healthy older adults and people with mild cognitive impairment: a systematic review. Ageing research reviews, 12(1), 263-275.

[9] Kueider, A. M., Parisi, J. M., Gross, A. L., \& Rebok, G. W. (2012). Computerized cognitive training with older adults: a systematic review. PloS one, 7(7), e40588.

[10] Kramer, A. F., \& Erickson, K. I. (2007). Capitalizing on cortical plasticity: influence of physical activity on cognition and brain function. Trends in cognitive sciences, 11(8), 342-348.

[11] Hillman, C. H., Erickson, K. I., \& Kramer, A. F. (2008). Be smart, exercise your heart: exercise effects on brain and cognition. Nature reviews neuroscience, $9(1), 58-65$.

[12] Langlois, F., Vu, T. T. M., Chassé, K., Dupuis, G., Kergoat, M. J., \& Bherer, L. (2013). Benefits of physical exercise training on cognition and quality of life in frail older adults. The Journals of Gerontology Series B: Psychological Sciences and Social Sciences, 68(3), 400-404.

[13] Curlik, D. M., \& Shors, T. J. (2013). Training your brain: do mental and physical (MAP) training enhance cognition through the process of neurogenesis in the hippocampus?. Neuropharmacology, 64, 506-514.

[14] Liu-Ambrose, T., \& Donaldson, M. G. (2009). Exercise and cognition in older adults: is there a role for resistance training programmes?. British journal of sports medicine, 43(1), 25-27.

[15] Baker, L. D., Frank, L. L., Foster-Schubert, K., Green, P. S., Wilkinson, C. W., McTiernan, A., ... \& Duncan, G. E. (2010). Effects of aerobic exercise on mild cognitive impairment: a controlled trial. Archives of neurology, 67(1), 71-79.

[16] Coelho, F. G. D. M., Andrade, L. P., Pedroso, R. V., SantosGalduroz, R. F., Gobbi, S., Costa, J. L. R., \& Gobbi, L. T. B. (2013). Multimodal exercise intervention improves frontal cognitive functions and gait in Alzheimer's disease: a controlled trial. Geriatrics \& gerontology international, 13(1), 198-203.

[17] Zimmermann, P., \& Fimm, B. (1995). Test battery for attention performance (TAP). Psytest, Wuerselen, Germany.

[18] Cavallo F., Maremmani C., Esposito D., Rovini E., Aquilano M., Dario P., Carrozza M.C., "Method and related apparatus for monitoring biomechanical performances of human limbs". Pct/ib2014/ 058265,14 jan 2014.

[19] Petersen, R. C. (2004). Mild cognitive impairment as a diagnostic entity. Journal of internal medicine, 256(3), 183-194.

[20] Rousseeuw, P. J. (1987). Silhouettes: a graphical aid to the interpretation and validation of cluster analysis. Journal of computational and applied mathematics, 20, 53-65. 